\title{
Aplicación del Láser de Baja Potencia (LLLT) en Pacientes Pediátricos: Revisión de Literatura a Propósito de una Serie de Casos
}

\author{
Application of Low Power Laser (LLLT) in Pediatric Patients: \\ Review of the Literature in a Series of Cases
}

\author{
Francisco Antonio Donoso Martínez ${ }^{1,5}$; Betty Bizcar²; Catalina Sandoval ${ }^{3}$ \& Paulo Sandoval Vidal ${ }^{4}$
}

DONOSO, M. F. A.; BIZCAR, B.; SANDOVAL, C. \& SANDOVAL, V. P. Aplicación del láser de baja potencia (LLLT) en pacientes pediátricos: revisión de literatura a propósito de una serie de casos. Int. J. Odontostomat., 12(3):269-273, 2018.

RESUMEN: El uso del láser de baja potencia en odontología está siendo cada vez más frecuente y con excelentes resultados, los equipos más modernos traen pre-establecidos la dosimetría de los parámetros para las diferentes acciones clínicas. El objetivo de esta revisión fue encontrar evidencia científica que permita al clínico tener como una alternativa de tratamiento el uso del láser de baja potencia en patologías orales de tejidos blandos y duros en pacientes pediátricos. Se realizó una revisión de la literatura por medio de buscadores como PubMed, LILACS y SciELO. Después de leer a texto completo todos los trabajos es posible señalar que el láser de baja frecuencia es una buena alternativa terapéutica en la resolución de signos y síntomas en patologías orales que aquejan al paciente pediátrico, puesto que, presenta un rápido control del dolor, la inflamación, el sangrado y acelera los procesos de reparación celular.

PALABRAS CLAVE: láser diodo, LLLT, frenectomía, láser odontología pediátrica, úlceras aftosas.

\section{INTRODUCCIÓN}

El láser de diodo presenta como medio activo un semiconductor de estado sólido compuesto de aluminio, arseniuro de galio y algunas ocasiones de indio. La longitud de onda van desde los 810-980 nanómetros aproximadamente. Sus ondas son invisibles al ojo humano. El láser de diodo presenta la conducción de sus ondas por medio de un cable de fibra óptica o a través de una punta óptica desechable. Estas ondas son absorbidas en su totalidad por el tejido pigmentado y por el contrario el tejido dentario presenta una mala absorción lo cual permite trabajar con tranquilidad a su alrededor (Hilgers \& Tracey, 2004). Está indicado en terapéuticas de remodelado gingival, retiro de tejido inflamatorio e hipertrófico, frenectomía, operculectomía, fenestración, fotoestimulación de lesiones traumáticas y post-quirúrgicas, tratamiento de aftas y herpes (Larrea-Oyarbide et al., 2004; Verma et al., 2012; Vale et al., 2015).

En relación al tratamiento de lesiones orales el grupo de investigación de Vale et al., hacen referencia, que durante los tratamientos de láser de baja frecuencia se liberan factores a nivel celular y sub-celular que favorecen la reparación tisular así como también el alivio del dolor. Señalan también no haber encontrado estudios que reporten efectos adversos, sin embargo, no reconocen la falta estudios con seguimiento a largo plazo.

\footnotetext{
${ }^{1}$ Estudiante Programa de Magister en Odontología, Facultad de Odontología, Universidad de La Frontera, Temuco, Chile.

${ }^{2}$ Magister en Odontología. Residente Programa de Especialidad en Ortodoncia y Ortopedia Dentomaxilar, Facultad de Odontología, Universidad de La Frontera, Temuco, Chile.

${ }^{3}$ Fonoaudióloga, Unidad de Ortodoncia Interceptiva, Facultad de Odontología, Universidad de La Frontera, Temuco, Chile.

${ }^{4}$ Director Programa de Especialidad en Ortodoncia y Ortopedia Dentomaxilar, Facultad de Odontología, Universidad de La Frontera, Temuco, Chile.

${ }^{5}$ Financiamiento de Conicyt. PFCHA/MagisterNacional/2017-22171665

Patrocinio: Programa de Magister en Odontología, Facultad de Odontología, Universidad de La Frontera, Temuco, Chile.
} 
Según lo reportado por Parker (2007), el cuidado más importante en la manipulación del láser de baja potencia es el riesgo de daño ocular para el operador, ayudante, personal auxiliar, así como para el paciente cuando se apunta directamente a la retina.

\section{MATERIAL Y MÉTODO}

Se llevó a cabo una revisión de la literatura por medio de buscadores como PubMed, LILACS y SciELO. El objetivo fue encontrar evidencia científica que permita al clínico tener como una alternativa de tratamiento el uso del láser de baja potencia en patologías de tejidos blandos orales en pacientes pediátricos. Los criterios fueron trabajos escritos en inglés, portugués y español, disponibilidad de texto completo, investigaciones con láser de baja potencia en relación al tipo de trabajo se seleccionaron reporte de caso, serie de casos, revisiones sistemáticas, estudios in vitro y estudios clínicos.

\section{RESULTADOS}

Uso del láser de baja frecuencia en el tratamiento de ulceras aftosas: En la revisión sistemática realizada por Vale et al. encontraron diferencias estadísticamente significativas entre los grupos experimental (láser de baja potencia) y el grupo control, en el tratamiento de ulceras aftosas recurrentes orales. Se midieron variables como dolor por medio de una escala visual análoga (EVA), dificultad para alimentarse, para cepillarse los dientes, tamaño de la lesión y tiempo de curación. En relación a los resultados encontrados señala que no es posible entregar un protocolo clínico de trabajo, pero en relación a los resultados encontrados si pueden sugerir el uso de láser de baja potencia como una alternativa de tratamiento de aftas. Sugiere hacer más estudios para tener un protocolo de trabajo y para establecer mecanismos más específicos por los cuales el láser de baja potencia promueve el alivio del dolor y la curación de las aftas. Lalabonova \& Daskalov (2014) realizaron un estudio clínico donde el grupo experimental $(n=90)$ se aplicó terapia de láser de baja potencia para el tratamiento de ulceras aftosas y el grupo control $(n=90)$ recibió terapia medicamentosa. Los parámetros medidos fueron dolor (EVA), eritema, tiempo de epitelización. Las variables fueron medidas el día 1, 2, 3 y 5 . Todas fueron estadísticamente significativas a partir del tercer día. De acuerdo con los resultados concluyen que el tratamiento con láser de baja potencia tiene más eficacia en comparación con la farmacoterapia en el alivio de signos y síntomas de las ulceras aftosas. Tellez Tielves et al. (2013) encontraron resultados similares en su estudio que mide las mismas variables del estudio antes mencionado. Los autores concluyen que la terapia con láser de baja potencia ofrece una mejor alternativa de tratamiento a esta patología que es tan recurrente y dolorosa (Fig. 1).

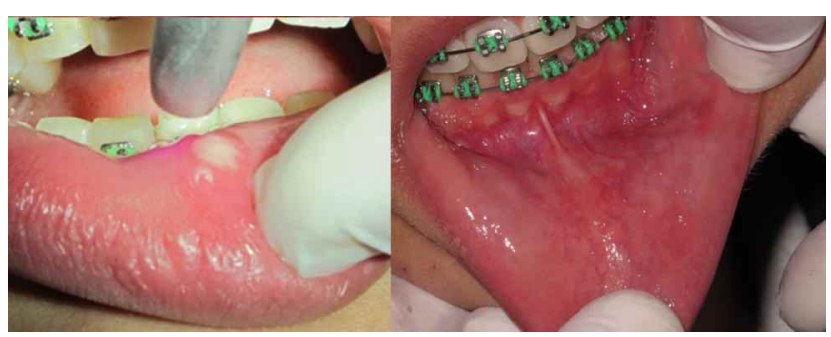

Fig. 1. Láser de baja potencia ofrece como alternativa al tratamiento farmacológico para las ulceras aftosas recurrentes orales.

Uso del láser de baja frecuencia en el tratamiento de herpes labial: El herpes simple (HSV-1) es una infección viral. Su incidencia llega al peak entre los 2 y 3 años. El virus permanece en las terminaciones nerviosas y establece un estado de latencia en los ganglios sensoriales del nervio trigémino. La reactivación puede ser causada por estímulos como el estrés, inmunodepresión, fiebre, entre otras, Stona et al. (2014) realizaron un reporte de un caso con el objetivo de evaluar el uso de láser de baja potencia (LLLT) como una opción de tratamiento en lesiones por herpes labial recurrente en odontología pediátrica. El caso reportado corresponde a un niño de 7 años, con dificultad para alimentarse, falta de apetito, vesículas y dolor en el bermellón del labio inferior, malestar general, dificultades para dormir. Al paciente se le realizaron 3 terapias con láser de baja potencia, una cada 24 horas. En la segunda aplicación el paciente ya no presentó sintomatología y las ampollas llegaron en estado de costra, pero asintomáticas. Al décimo día la lesión del labio inferior llegó casi recuperada. Concluyen que la terapia con láser de baja potencia es una alternativa a considerar en el tratamiento de herpes labial en odontología pediátrica, mostrando un rápido alivio del dolor y acelerada reparación tisular. Ferreira et al. (2011) reportaron dos casos de adultos (23 y 50 años) tratados con láser de baja potencia. El objetivo era realizar un seguimiento a largo plazo luego de realizado el tratamiento. En ambos casos se realizaron 4 sesiones. Al quinto día las lesiones remitieron com- 
pletamente. El seguimiento fue realizado hasta los 17 meses post-tratamiento, periodo durante el cual no se observó recurrencia. Los autores concluyen que es una buena alternativa de tratamiento en pacientes que rechazan tratamiento medicamentoso, mientras que se está a la espera de una vacuna anti-herpética (Fig. 2).
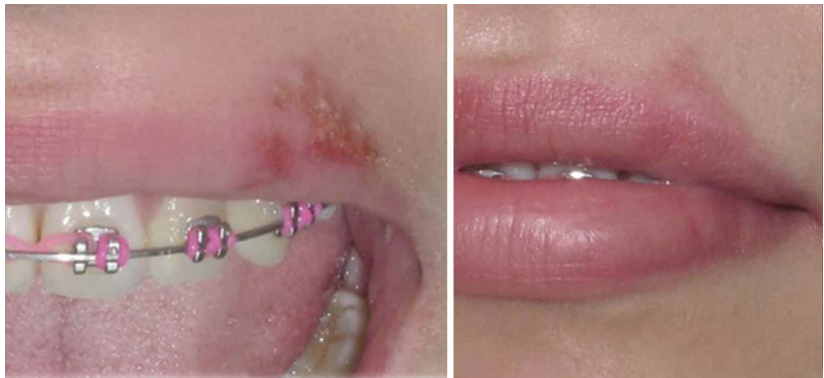

Fig. 2. Herpes labial tratado con Láser de baja potencia. Antes y después de 5 días.

Uso del láser en frenectomía: Gargari et al. (2012) realizan el reporte de un caso de frenectomía labial inferior, realizada con láser de baja potencia. Paciente con tracción del margen gingival por acción continua de frenillo, se usa láser de diodo de $940 \mathrm{~nm}$, lidocaína spray al $10 \%$, aspiración del vapor durante el corte. Ausencia de dolor y sangrado durante el procedimiento. Al día 21 presentó continuidad de los teji- dos con ausencia de cicatriz. Los autores concluyen que el láser de diodo presenta ventajas sobre la técnica convencional en relación a la hemostasia, precisión en el corte, mínimo dolor e inflamación postoperatoria. Kafas et al. (2009) reportaron un caso de frenectomía labial superior en un niño de 9 años de edad. Se aplicó spray de lidocaína, no se usó anestesia infiltrativa, el corte incluyó el frenillo más una extensión hacia palatino pasando entre los incisivos. El paciente no refirió dolor durante ni tampoco después del procedimiento. Se controló el paciente, y a los diez días su reparación se consideró óptima. Se concluye que el láser es una herramienta útil para el clínico en la frenectomía labial pediátrica (Fig. 3).

Otra aplicación es para fenestrar dientes en erupción retrasada, la que tradicionalmente se realiza con bisturí y que con láser resulta en un procedimiento menos sangriento, y con una reparación más rápida, pero por sobre todo más aceptable para los niños (Fig. 4).

Para exéresis de lesiones de tejidos blandos, el láser reemplaza al bisturí en su capacidad de cortar. Muchas pequeñas lesiones pueden ser eliminadas mediante una alta energía y con los tips activados como se ejemplifica en la figura 5 donde se eliminó un mucocele recurrente (Fig. 5).
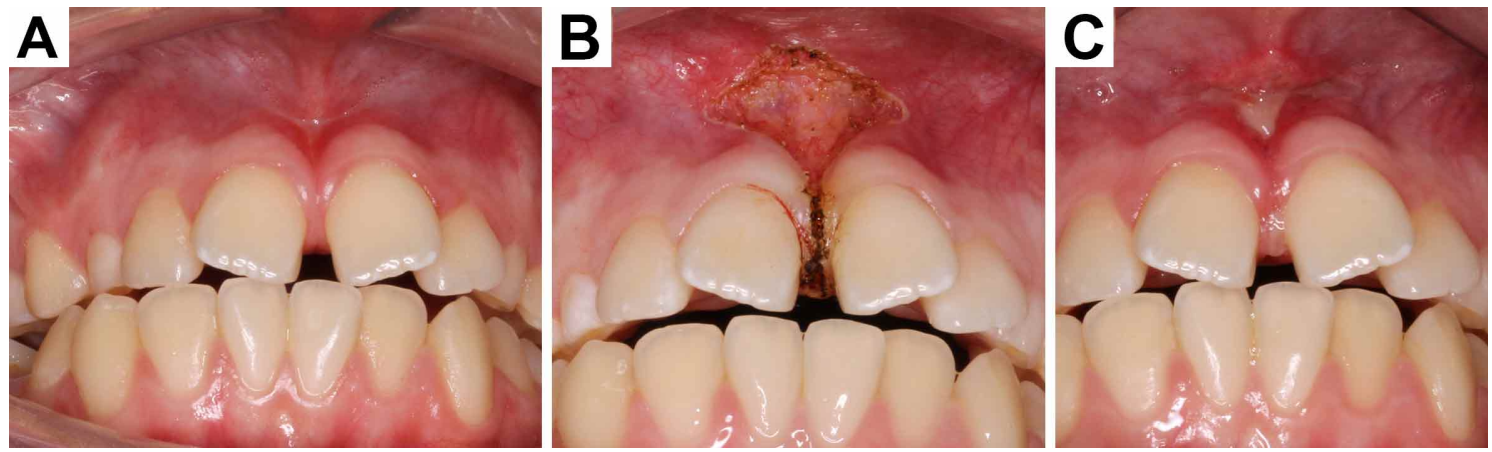

Fig. 3. Frenectomía realizada con Láser de diodo. a) Antes, b) al finalizar el procedimiento. c) Control siete días después.

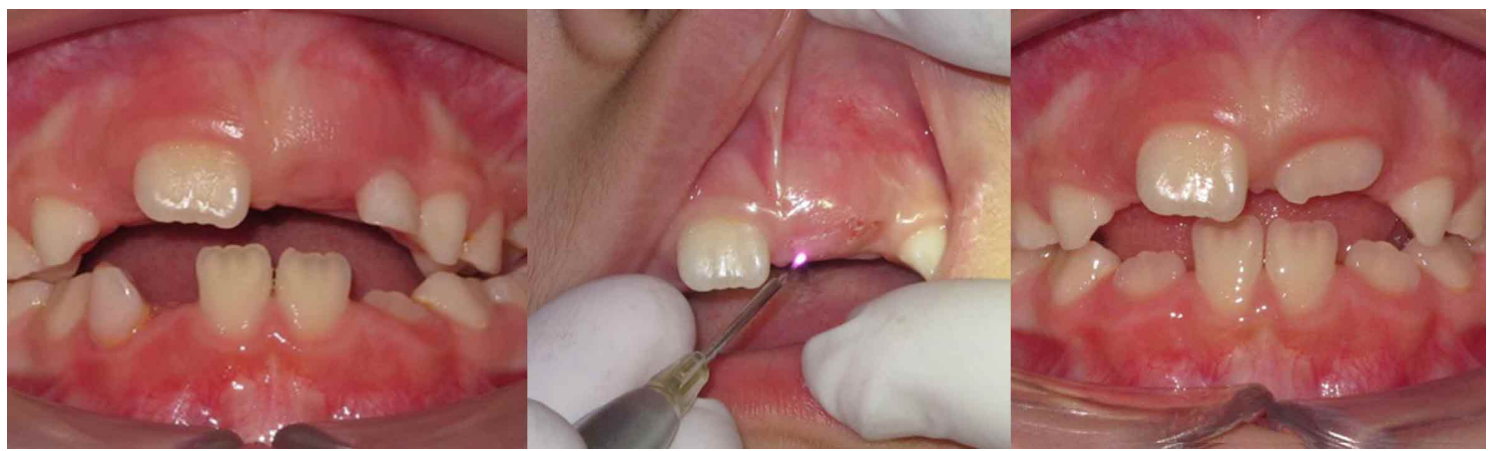

Fig. 4. Fenestración de encía que permite la erupción dental asimétrica realizada con Láser de diodo. 


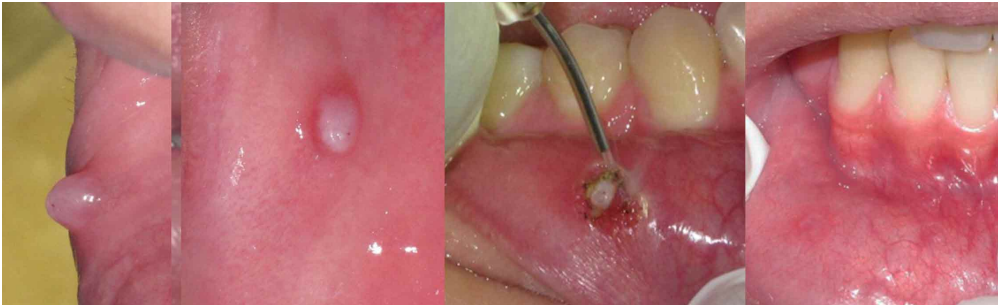

Fig. 5. Retiro de mucocele con Láser de diodo y control siete días después.

Uso del láser de baja potencia en ortodoncia y ortopedia dentofacial. Cepera et al. (2012) evaluó el efecto de la aplicación de láser de baja potencia en regeneración ósea en procesos de expansión palatina rápida, para esto estudió 27 niños de 8 a 12 años de edad, todos con indicación de expansión palatina rápida y los dividió en 2 grupos, con aplicación de láser $(n=14)$ y sin la aplicación de láser $(n=13)$ utilizando un láser con una longitud de onda de $780 \mathrm{~nm}, 40 \mathrm{~mW}$ de potencia y y 10 $\mathrm{J} / \mathrm{cm}^{2}$ de fluencia, en 10 puntos localizados alrededor de la sutura palatina media según protocolo de aplicación definido por los investigadores, concluyendo que el láser de baja potencia, asociado con la rápida expansión maxilar, proporciona una apertura eficiente de la sutura palatina media e influye en el proceso de regeneración ósea de la sutura, acelerando la cicatrización.

García et al. (2016) estudiaron el efecto del láser terapéutico en la reparación y regeneración ósea en dos grupos de pacientes de 6 a 12 años de edad, después de realizar disyunción media palatina (Fig. 6). Concluyendo luego de 75 días de la fase de retención, que la sutura media palatina presenta diferentes niveles de reorganización según el área analizada. LLLT parece estimular el proceso de reparación durante la fase de retención después de RME.

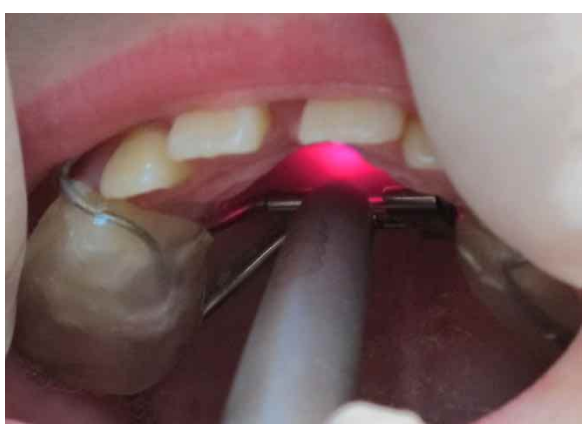

Fig. 6. Disyunción media palatina fotobioestimulada con Láser.

Stein et al. (2015) de la Universidad de Philipps, Marburg, Alemania, investigaron si la terapia con láser de baja potencia
(LLLT) tenía un efecto reductor del dolor en 40 pacientes pediátricos de edad media 8,05 años, sometidos a separaciones molares de uso ortodóncico. El estudio evaluó un grupo de 20 pacientes cuyo tratamiento incluyó la aplicación de láser de diodo de baja potencia $(660 \mathrm{~nm}, 100 \mathrm{~mW})$, el día de la inserción de la separación molar y un grupo control de 20 pacientes que no recibían LLLT. La percepción del dolor se redujo significativamente ( $p$ $<0,05$ ) en el grupo LLLT el día 1 y se continuó reduciendo el día 2 en comparación con el grupo control. Dado sus hallazgos es posible esperar un efecto reductor del dolor en pacientes pediátricos sometidos a separaciones molares en dentición mixta temprana, LLLT es una alternativa interesante de proporcionar analgesia incluso en pacientes muy jóvenes.

La aplicación múltiple es posible en niños, lo cual se ejemplifica en el último caso. Un paciente de 8 años se presenta con un incisivo desalineado pero que en la radiografía panorámica muestra que el otro incisivo viene con el mismo problema (Figs. 7a y 7b). Se inicia una terapia convencional con aparatos fijos, sin embargo para que erupcione el diente 2.1 se realiza una operculectomía con láser y se aprecia una giroversión de más de 90 grados (Fig. 7c). Se inicia una terapia complementaria con láser tanto para bioestimular a las células del ligamento periodontal además de las fuerzas ligeras en cupla. A los nueve meses de tratamiento se realiza una frenectomía del labio superior (Fig. 7d). Cinco meses después se logra el alineamiento de ambos incisivos y se mantiene por un año con aparatos para que el hueso se remodele completamente (Fig. 7e). Finalmente se realiza una gingivectomía con láser del diente 1.1 para homogenizar el margen gingival (Fig. 7f).
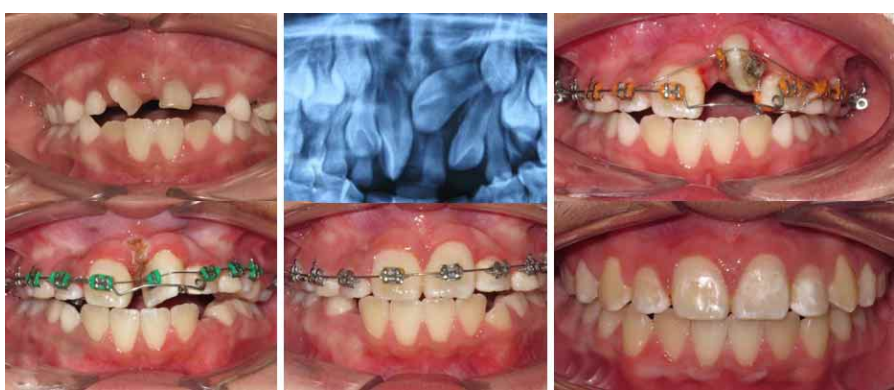

Fig. 7. a) Presencia de incisivo temporal (diente 6.1) persistente, b) Radiografía mostrando diente 2.1 en erupción anómala, c) Rotación con aparatos fijos post-operculectomía láser, d) Frenectomía central con láser de diodo, e) Alineación diente 2.1 fotobioestimulado mensualmente con láser de baja frecuencia, f) Post-gingivectomía en dientes 11 y 12 con láser de diodo para alinear el margen gingival al finalizar el tratamiento con braquets de ortodoncia. 


\section{CONCLUSIÓN}

El láser de baja potencia es una buena alternativa terapéutica en la resolución de signos y síntomas en patologías orales que aquejan al paciente pediátrico, puesto que, presenta un rápido control del dolor, la inflamación y el sangrado, además acelera los procesos de reparación celular, es silencioso y no asusta a los niños. Sin embargo son necesarios más estudios que permitan saber cuál es la estabilidad de los tratamientos a largo plazo y poder establecer con mayor precisión los mecanismos celulares y moleculares involucrados en la reparación acelerada de los tejidos.

DONOSO, M. F. A.; BIZCAR, B.; SANDOVAL, C. \& SANDOVAL, V. P. Application of low power laser (LLLT) in pediatric patients: Review of the literature in a series of cases. Int. J. Odontostomat., 12(3):269-273, 2018.

ABSTRACT: Low Level Laser Therapy in dentistry is becoming more frequent and has had excellent results, with state of the art equipment having pre-established dosimetry parameters for the various clinical actions. The objective of this review was to find scientific evidence that allows the clinician to have the use of low power laser as treatment alternative, in oral soft tissue and hard pathologies in pediatric patients. A review of the literature was performed through search engines such as PubMed, LILACS and SciELO. After reading the texts, it was possible to point out that low frequency laser is a good therapeutic alternative in the resolution of signs and symptoms in oral pathologies of the pediatric patient. This alternative allows for rapid pain control, reduces inflammation and bleeding, and accelerates cellular repair processes.

KEY WORDS: Laser diode, LLLT, frenectomy, laser pediatric dentistry, aphthous ulcers.

\section{REFERENCIAS BIBLIOGRÁFICAS}

Cepera, F.; Torres, F. C.; Scanavini, M. A.; Paranhos, L. R.; Capelozza Filho, L.; Cardoso, M. A.; Siqueira, D. C. \& Siqueira, D. F. Effect of a low-level laser on bone regeneration after rapid maxillary expansion. Am. J. Orthod. Dentofacial Orthop., 141(4):444-50, 2012.

Ferreira, D. C.; Reis, H. L.; Cavalcante, F. S.; Santos, K. R. \& Passos, M. R. Recurrent herpes simplex infections: laser therapy as a potential tool for long-term successful treatment. Rev. Soc. Bras. Med. Trop., 44(3):397-9, 2011.

García, V. J.; Arnabat, J.; Comesaña, R.; Kasem, K.; Ustrell, J. M.; Pasetto, S.; Segura, O. P.; Manzanares-Céspedes, M. C. \&
Carvalho-Lobato, P. Effect of low-level laser therapy after rapid maxillary expansion: a clinical investigation. Lasers Med. Sci., 31(6):1185-94, 2016.

Gargari, M.; Autili, N.; Petrone, A. \& Prete, V. Using the diode laser in the lower labial frenum removal. Oral Implantol. (Rome), 5(23):54-7, 2012.

Hilgers, J. J. \& Tracey, S. G. Clinical uses of diode lasers in orthodontics. J. Clin. Orthod., 38(5):266-73, 2004.

Kafas, P.; Stavrianos, C.; Jerjes, W.; Upile, T.; Vourvachis, M.; Theodoridis, M. \& Stavrianou, I. Upper-lip laser frenectomy without infiltrated anaesthesia in a paediatric patient: a case report. Cases J., 2:7138, 2009.

Lalabonova, H. \& Daskalov, H. Clinical assessment of the therapeutic effect of low-level laser therapy on chronic recurrent aphthous stomatitis. Biotechnol. Biotechnol. Equip., 28(5):929-33, 2014.

Larrea-Oyarbide, N.; España-Tost, A. J.; Berini-Aytés, L. \& Gay-Escoda, C. Aplicaciones del láser de diodo en Odontología. R. C. O. E., 9(5):529-34, 2004.

Parker, S. Laser regulation and safety in general dental practice. $\mathrm{Br}$. Dent. J., 202(9):523-32, 2007.

Stein, S.; Korbmacher-Steiner, H.; Popovic, N. \& Braun, A. Pain reduced by low-level laser therapy during use of orthodontic separators in early mixed dentition. J. Orofac. Orthop., 76(5):4319, 2015.

Stona, P.; da Silva Viana, E.; Dos Santos Pires, L.; Blessmann Weber, J. B. \& Floriani Kramer, P. Recurrent labial herpes simplex in pediatric dentistry: Low-level laser therapy as a treatment option. Int. J. Clin. Pediatr. Dent., 7(2):140-3, 2014.

Tellez Tielves, N. C.; Pérez Hernández, L. Y.; Rodríguez Ávila, J.; Travieso Pérez, M. \& López Blanco, M. N. Efectividad del tratamiento con radiación láser de baja potencia en la estomatitis aftosa recurrente. Rev. Cienc. Med., 17(5):40-50, 2013.

Vale, F. A.; Moreira, M. S.; de Almeida, F. C. \& Ramalho, K. M. Lowlevel laser therapy in the treatment of recurrent aphthous ulcers: a systematic review. ScientificWorldJournal, 2015:150412, 2015.

Verma, S. K.; Maheshwari, S.; Singh, R. K. \& Chaudhari, P. K. Laser in dentistry: An innovative tool in modern dental practice. Natl. J. Maxillofac. Surg., 3(2):124-32, 2012.

Dirección para correspondencia:

Paulo Sandoval

Departamento de Pediatría y Ortodoncia

Universidad de La Frontera

Temuco

CHILE

E-mail: paulo.sandoval@ufrontera.cl

Recibido : 14-04-2018

Aceptado: 01-06-2018 